\section{Manejo perioperatório de pacientes em uso de anticoagulantes orais}

0 manejo perioperatório de pacientes em uso regular de anticoagulantes orais considera o risco de tromboembolia e das complicações hemorrágicas associado a diferentes estratégias de anticoagulação.(D) Enquanto o risco de sangramento depende fundamentalmente do local e do tipo de cirurgia, o risco de tromboembolia está ligado à indicação prévia para o uso regular de anticoagulantes (profilaxia arterial ou venosa), ao tempo decorrido desde o episódio de trombose e ao tipo de procedimento a ser realizado. Além disso, deve-se considerar se a cirurgia será realizada em caráter eletivo ou de urgência. ${ }^{(1)} 0$ Quadro 20 descreve esses contextos.

Para pacientes em uso regular de anticoagulantes orais, sempre que possivel, o procedimento cirúrgico deve ser realizado em caráter eletivo, para que haja tempo suficiente para reverter a anticoagulação.(D) ${ }^{(2)}$ Em pacientes cuja RNI está no nível terapêutico (geralmente 2-3), interrompe-se o uso do anticoagulante oral 5 dias antes, obtendo-se nova RN1 no dia anterior à cirurgia, o qual deve estar menor que 1,5. ldade avançada, insuficiência cardíaca congestiva descompensada, câncer e RNI muito elevada são fatores que retardam o retorno espontâneo de $\mathrm{RN1}$ a níveis próximos da normalidade.(C) ${ }^{(3)}$ Nos pacientes com alto risco de trombose, deve-se fazer um estratégia de transição, iniciando HBPM (paciente ambulatorial ou hospitalizado) ou HNF em infusão contínua (indivíduo hospitalizado) quando a RN1 estiver subterapêutica.(B) A suspensão das heparinas deve ser de $6 \mathrm{~h}$ antes da cirurgia para a infusão de HNF e de 12-24 h antes para a última dose de HBPM. Em procedimentos odontológicos, os pacientes podem ser operados com RN1 na faixa terapêutica e serem utilizados agentes hemostáticos tópicos (por ex., ácido tranexâmico).(C) ${ }^{(4)}$

A anticoagulação deve ser reiniciada, normalmente na dose pré-operatória, assim que a hemostasia esteja assegurada, e isso deve ser sempre discutido com a equipe cirúrgica, em geral, $12 \mathrm{~h}$ após o procedimento.(D) A reintrodução do anticoagulante deverá ser retardada nos casos de neurocirurgia e naqueles pacientes que permaneçam com sangramento ativo. Nos casos de alto risco de trombose, os pacientes devem ser mantidos com heparina até que a RNI alvo do anticoagulante oral seja atingida. ${ }^{(5)}$

Em procedimentos cirúrgicos que necessitam ser realizados em 24-96 h, pode-se administrar vitamina $\mathrm{K}$ oral (2-3 $\mathrm{mg}$ - a própria ampola de medicação i.v./s.c., já que é fabricada com micelas mistas que permitem a absorção intestinal).(B) Essa conduta faz a RN1 reduzir mais rapidamente sem causar uma resistência ao uso do anticoagulante oral no pós-operatório, que pode perdurar por até 1 semana. ${ }^{(6)}$ Após o uso da vitamina K, a RNI deve ser repetida a cada 8-24 h dependendo do nível inicial, já que a medicação pode ser usada novamente. ${ }^{(7)}$

Nos casos de urgência cirúrgica (nas próximas $24 \mathrm{~h}$ ), deve-se suspender 0 anticoagulante oral e administrar vitamina $\mathrm{K}$ i.v., concentrado de complexo protrombínico ou plasma fresco. $(B)^{(8)}$ Em casos específicos, o fator recombinante Vlla pode ser considerado, mediante consulta ao hematologista. ${ }^{(1)}$ A RN1 deve ser monitorada amiúde.

Outro aspecto essencial no manejo perioperatório de pacientes em uso de anticoagulantes é a necessidade de bloqueio neuroaxial (anestesia espinhal, anestesia epidural ou analgesia epidural contínua). Nos pacientes cirúrgicos, essas são eficazes no controle da dor, permitem a deambulação precoce do paciente e reduzem as taxas de complicações pulmonares. 0 hematoma periespinhal é uma rara complicação da anestesia epidural, com incidência estimada em menos de 1:150.000 pacientes em anestesias epidurais e em menos de 1:220.000 pacientes em anestesias espinhais, mas com consequências potencialmente graves. ${ }^{(9)} \mathrm{Na}$ maioria dos casos descritos, houve uma combinação de fatores de risco, envolvendo a inserção traumática de agulhas e de cateteres, anormalidades anatômicas ou vasculares da coluna lombar, uso de anticoagulantes e coagulopatias. Nas formas típicas, os pacientes apresentam dor na região dorsal acompanhada de fenômenos sensitivos e motores. 0 sangramento no espaço restrito do canal medular comprime e causa isquemia às fibras nervosas, podendo resultar em paraplegia, se não for realizada laminectomia descompressiva dentro das próximas $8 \mathrm{~h}$.

AAssociação Americana de Anestesia Regional elaborou recomendações para essa situação. ${ }^{(10)}$ Pacientes em uso de fármacos que atuam nos fatores da coagulação e na função plaquetária 
Quadro 20 - Riscos de sangramento e tromboembolia em diferentes contextos cirúrgicos.

\begin{tabular}{|c|c|}
\hline Risco de sangramento & Risco de tromboembolia \\
\hline Alto & Alto \\
\hline $\begin{array}{l}\text { - Todas as cirurgias de grande porte e aquelas que } \\
\text { envolvam a abertura de cavidades }\end{array}$ & $\begin{array}{l}\text { - Próteses de válvula mitral ou os modelos antigos } \\
\text { de próteses aórticas }\end{array}$ \\
\hline $\begin{array}{l}\text { - Procedimentos transcutâneos com agulhas em áreas } \\
\text { não compressíveis, incluindo biópsias viscerais }\end{array}$ & $\begin{array}{l}\text { - Fibrilação atrial + passado de AVC ou risco } \\
\text { aumentado adicional para AVC }\end{array}$ \\
\hline - Qualquer tipo de cirurgia de próstata & - TEP/TVP nos últimos 3 meses \\
\hline $\begin{array}{l}\text { - Cirurgias em locais específicos, em que pequenos } \\
\text { sangramentos costumam acarretar grande morbidade, } \\
\text { como no sistema nervoso central e em certos } \\
\text { procedimentos oftálmicos }\end{array}$ & $\begin{array}{l}\text { - Estados de hipercoagulabilidade com episódio } \\
\text { trombótico recente, trombose recorrente ou história } \\
\text { de trombose com risco de vida }\end{array}$ \\
\hline Baixo & Baixo \\
\hline $\begin{array}{l}\text { - Procedimentos transcutâneos com agulhas, em } \\
\text { áreas facilmente compressiveis }\end{array}$ & $\begin{array}{l}\text { - Modelos mais recentes de próteses aórticas ou } \\
\text { quaisquer modelos de próteses biológicas }\end{array}$ \\
\hline - A maioria dos procedimentos cutâneos & $\begin{array}{l}\text { - Fibrilação atrial sem outros fatores de risco para } \\
\text { AVC }\end{array}$ \\
\hline \multirow{2}{*}{$\begin{array}{l}\text { - Procedimentos dentários de rotina (limpeza, } \\
\text { restaurações, intervenções endodônticas, próteses e } \\
\text { extrações simples) }\end{array}$} & - TEP/TVP há mais de 3 meses \\
\hline & $\begin{array}{l}\text { - Estados de hipercoagulabilidade sem episódio } \\
\text { trombótico recente, trombose recorrente ou passado } \\
\text { de trombose com risco de vida }\end{array}$ \\
\hline
\end{tabular}

são os principais candidatos às complicações associadas a esses procedimentos. A anestesia e a analgesia neuroaxiais devem ser evitadas em pacientes com discrasias sanguíneas, bem como naqueles em uso de drogas antitrombóticas. São contraindicações para a anestesia e a analgesia neuroaxiais $\mathrm{RNI}>1,5$, tempo de protrombina $>1,5$ vezes o controle e contagem plaquetária $<50.000 / \mathrm{mm}^{3}$.(D)

0 uso de anti-inflamatórios não esteroides e de ácido acetilsalicílico provavelmente não aumenta isoladamente o risco de hematoma epidural. Apesar da inexistência de estudos randomizados que avaliem o risco de hematoma epidural associado ao uso de clopidogrel e ticlopidina, recomenda-se sua interrupção 5-14 dias antes do procedimento.(C) ${ }^{(1)}$ Em pacientes em uso de anticoagulantes no pré-operatório, a inserção de agulha ou cateter no canal medular deve ser retardada, até que o efeito anticoagulante seja revertido. 1sso costuma ocorrer depois de, pelo menos, 8-12 h após uma dose de HNF s.c. ou de HBPM s.c. quando empregada em duas doses diárias. Considerando-se o esquema posológico caracterizado por uma dose diária de HBPM, deve-se observar um período mínimo de $18 \mathrm{~h}$. Para o esquema com HBPM (2 vezes ao dia), a primeira dose é habitualmente ministrada 12-24 h depois da cirurgia, e a remoção do cateter epidural é recomendada antes do início da tromboprofilaxia. Se o cateter epidural for mantido no local durante a tromboprofilaxia com HBPM (duas vezes ao dia), um retardo de $24 \mathrm{~h}$, entre a última dose de HBPM e a remoção do cateter, é recomendado.(B) ${ }^{(10)}$

Cateteres epidurais somente devem ser removidos quando o efeito anticoagulante for desprezível. Se a profilaxia com anticoagulantes orais for inevitável, recomenda-se não manter o cateter instalado por mais de 1-2 dias, devido à sua imprevisibilidade. Nesses casos, a RN1 deve ser mantida < 1,5 no momento da remoção do cateter.(C) $)^{(11,12)}$

Outros cuidados em pacientes sob medicação anticoagulante são os seguintes: a via intramuscular deve ser evitada; se for inserido um cateter venoso central, deve-se preferir um local de fácil compressão (por ex., evitar a punção de veia subclávia); não inserir intubação nasotraqueal; e não instalar sonda nasogástrica.

\section{Referências}

1. Douketis JD, Berger PB, Dunn AS, Jaffer AK, Spyropoulos $\mathrm{AC}$, Becker RC, et al. The perioperative management of antithrombotic therapy: American College of Chest Physicians Evidence-Based Clinical Practice Guidelines (8th Edition). Chest. 2008;133(6 Suppl):299S-339S.

2. Bombeli T, Spahn DR. Updates in perioperative coagulation: physiology and management of 
thromboembolism and haemorrhage. $\mathrm{Br} \mathrm{J}$ Anaesth. 2004;93(2):275-87.

3. Hylek EM, Regan S, Go AS, Hughes RA, Singer DE, Skates SJ. Clinical predictors of prolonged delay in return of the international normalized ratio to within the therapeutic range after excessive anticoagulation with warfarin. Ann Intern Med. 2001;135(6):393-400.

4. Blinder D, Manor Y, Martinowitz U, Taicher S, Hashomer T. Dental extractions in patients maintained on continued oral anticoagulant: comparison of local hemostatic modalities. Oral Surg Oral Med Oral Pathol Oral Radiol Endod. 1999;88(2):137-40.

5. Kearon C, Hirsh J. Management of anticoagulation before and after elective surgery. $\mathrm{N}$ Engl J Med. 1997;336(21):1506-11.

6. Hirsh J, Fuster V, Ansell J, Halperin JL; American Heart Association; American College of Cardiology Foundation. American Heart Association/American College of Cardiology Foundation guide to warfarin therapy. Circulation. 2003;107(12):1692-711.
7. Dunn AS, Turpie AG. Perioperative management of patients receiving oral anticoagulants: a systematic review. Arch Intern Med. 2003;163(8):901-8.

8. 0'Shaughnessy DF, Atterbury C, Bolton Maggs P, Murphy M, Thomas D, Yates S, et al. Guidelines for the use of fresh-frozen plasma, cryoprecipitate and cryosupernatant. Br J Haematol. 2004;126(1):11-28.

9. Horlocker TT. Low molecular weight heparin and neuraxial anesthesia. Thromb Res. 2001;101(1):V14154.

10. Horlocker TT, Wedel DJ, Benzon H, Brown DL, Enneking FK, Heit JA, et al. Regional anesthesia in the anticoagulated patient: defining the risks (the second ASRA Consensus Conference on Neuraxial Anesthesia and Anticoagulation). Reg Anesth Pain Med. 2003;28(3):172-97.

11. Tyagi A, Bhattacharya A. Central neuraxial blocks and anticoagulation: a review of current trends. Eur J Anaesthesiol. 2002;19(5):317-29.

12. Institute for Clinical Systems Improvement. Venous thromboembolism prophylaxis. Bloomington: Institute for Clinical System Improvement; 2009. 